\title{
Proyeccion Social de Derecho: PROSODE, un aporte de proyección y responsabilidad social
}

\author{
A don Carlos Montoya Anguerry, por su ejemplo \\ en la búsqueda de justicia, solidaridad y ética ${ }^{1}$. \\ A PROSODE, fuente de aprendizaje significativo.
}

\section{INTRODUCCIÓN}

Se me planteó escribir un artículo sobre el aporte de PROSODE hacia la responsabilidad social en nuestra Facultad, una tarea que acepté con mucha alegría. Pero es pertinente precisar que este no podrá ser enteramente neutral, pues me siento profundamente comprometido y conectado con la experiencia de proyección y responsabilidad social que ha provocado PROSODE en la Facultad de Derecho y en la comunidad.

Formo parte de este programa desde que fui alumno del curso Problemas Jurídicos de los Sectores Marginales ${ }^{2}$ de la Facultad de Derecho durante el semestre 1992-1, en el consultorio jurídico de la parroquia La Recoleta, y debo decir que el curso impactó en mí y en muchos compañeros, lo que me motivó a continuar como colaborador, luego como jefe de práctica, después como profesor y, actualmente como profesor coordinador.

Con estas consideraciones, paso a desarrollar la experiencia de proyección social y responsabilidad social del curso Proyección Social PROSODE de nuestra Facultad de Derecho.

\section{RESPONSABILIDAD SOCIAL UNIVERSITARIA ${ }^{3}$}

La responsabilidad social empresarial (RSE) es un conjunto de prácticas de la organización que forman parte de su estrategia corporativa y que, siguiendo fines racionales, tiene como objetivo producir beneficios y/o evitar daños para todas las partes interesadas en la actividad de la empresa (clientes, empleados, accionistas, comunidad, entorno, etcétera). Debe, además, redondear en un beneficio tanto para la organización como para la sociedad.

* Informe elaborado por Iván Ortiz, profesor coordinador de PROSODE.

1 El profesor Montoya es el fundador de las acciones del curso de PROSODE. Fue quien institucionalizó, en la Facultad de Derecho, este programa.

2 Así se llamó inicialmente el curso que, desde el semestre 1993-1, se conoce como Proyección Social - PROSODE.

3 En esta sección hemos resumido libremente las ideas del ensayo «La responsabilidad social de la universidad" de François Vallaeys, publicado en el portal de asuntos públicos de la PUCP, Palestra, en http://www.palestra.pucp.edu.pe/index.php?id=111, y consultado el 12 de abril de 2005. Es importante mencionar que François Vallaeys y el profesor Luis Bacigalupo fueron los impulsores del enfoque de responsabilidad social universitaria en la PUCP. 
La responsabilidad social universitaria (RSU), que se deriva de la RSE, exige articular las diversas partes de la institución en un proyecto de promoción social de principios éticos y de desarrollo social equitativo y sostenible, para la producción y transmisión de saberes responsables y para la formación de profesionales ciudadanos igualmente responsables.

François Vallaeys plantea cuatro líneas de acción institucional de la responsabilidad social universitaria:

- Gestión interna. La meta es orientarla hacia la transformación de la universidad en un pequeña comunidad ejemplar de democracia, respetos de los derechos laborales, equidad, transparencia, y hacer de ella un modelo de desarrollo sostenible. Hacer de la universidad una comunidad socialmente ejemplar es beneficiarse de una doble fuente de aprendizaje: el estudiante aprende dentro de ella, pero también aprende de ella hábitos y valores ciudadanos.

- Docencia. La idea es capacitar a los docentes en el enfoque de la responsabilidad social universitaria y promover, en las especialidades, el aprendizaje basado en proyectos de carácter social ${ }^{4}$, y así abrir el salón de clases hacia la comunidad social como fuente de enseñanza significativa y práctica aplicada a la solución de problemas reales. Se trata de ser creativos y de imaginar cómo el estudiante puede aprender haciendo cosas socialmente útiles y formarse como ciudadano informado y responsable.

- Investigación. La meta es promover la investigación para el desarrollo bajo todas las formas posibles. Por ejemplo, una estrategia posible sería que la universidad firme convenios de hermanamiento con distritos urbano-marginales o rurales e invite a los departamentos de diversas carreras a desarrollar investigaciones interdisciplinarias aplicadas con dichas localidades.

- Proyección social. La meta es trabajar en interfaz con los departamentos de investigación y con los docentes de las diversas facultades para implementar y administrar proyectos de desarrollo que puedan ser fuente de investigación aplicada y de recursos didácticos para la comunidad universitaria.

Consideramos que nuestra Facultad de Derecho, a través del curso Proyección Social - PROSODE, ha aportado nítida, clara y exitosamente en las líneas de docencia y proyección social planteadas por Vallaeys, y que condensa los ejes planteados en su concepto. 


\section{PROYECCIÓN SOCIAL DEL DERECHO - PROSODE ${ }^{5}$}

\section{II.1. Proyección social}

«Proyectar» es, según el diccionario de la Real Academia Española, «lanzar, dirigir hacia delante o a distancia» ${ }^{6}$. «Proyección» es una tarea que supone un plan para llevar a cabo en el tiempo, una operación permanente, una acción por emprender desde aquí y ahora hacia delante. «Social» implica que estas acciones y este lanzamiento recaigan inevitablemente sobre la comunidad en general, y sin exclusiones a priori de clase alguna, sin que ello signifique que dejemos de priorizar sectores determinados de acuerdo a los objetivos que se planteen y conforme a la realidad y a la cultura de cada país.

En ese sentido, considero que la proyección social del Derecho genera aprendizajes de un especial carácter dual. Consiste en «dar» $\mathrm{y}$ «recibir» complementariamente. Se actúa «dando» o brindando el servicio o la actividad en favor del grupo social beneficiario, pero al mismo tiempo se actúa «recibiendo» aprendizajes y/o experiencias (retorno) a partir de la ejecución o de la aplicación de la actividad o del servicio legal? ${ }^{7}$. Y es en ese sentido que nos encontramos también inmersos dentro de la metodología del aprendizaje-servicio.

Desde la perspectiva del Derecho, nosotros tenemos un deber con nuestra comunidad, pero no debemos perder de vista nuestra esencia como universidad: la formación integral y humanística de nuestros estudiantes y el rol de aprendizaje que también debe impulsar la proyección social. Por ello, el curso promueve el aprendizaje mediante una acción universitaria en la comunidad. Ello significa que la proyección social del Derecho prioriza la ejecución de actividades, servicios o proyectos sobre la población que atiende y que retorna en aprendizaje y conocimiento para nuestro estudiantes y docentes. Este es el real sentido de la responsabilidad social universitaria, pues la idea es actuar en la comunidad y que esta acción retorne a la comunidad universitaria como fuente de aprendizaje.

\section{II.2. Antecedentes y formación de PROSODE}

PROSODE se institucionaliza dentro de un proceso participativo y horizontal de la Facultad de Derecho que revisó el plan de estudios y el perfil profesional (Claustro Pleno) en $1989^{8}$.

5 Para esta sección, se ha tomado como base la sistematización sobre PROSODE denominada «Procesos de retorno de la proyección social a la PUCP según modelo de PROSODE», publicada en ORTIZ, Iván (coordinador). Proyección social a través del Derecho y de PROSODE. 15 años proyectando el Derecho a la comunidad. Lima: PUCP, 2006.

6 CISNEROS, Luis Jaime. El ámbito y el sentido de la proyección social, 1994.

7 Esta afirmación es coincidente con la del doctor Antonio Peña Jumpa en su ensayo «La proyección social del Derecho en un contexto pluricultural: perspectivas desde el caso peruano", publicado en Proyección social del Derecho y clínicas jurídicas en las universidades de América del siglo XXI. Madrid: Editorial Dickynson, 2005.

8 Luego del Claustro Pleno, se formó una Comisión de Proyección Social, presidida por el profesor Juan Luis Avendaño y conformada por Walter Albán Peralta, actual decano, y por la profesora

PROYECCION

SOCIAL DE DERECHO: PROSODE, UN APORTE DE PROYECCIÓN Y RESPONSABILIDAD SOCIAL 
En el semestre 1991-1 nace como curso bajo la dirección del profesor Carlos Montoya Anguerry, quien lo implementó como un curso electivo (voluntario) de tres créditos, denominado Problemas Jurídicos de los Sectores Marginales. En 1993, el curso cambió de nombre a Proyección Social de Derecho, más conocido por sus siglas, PROSODE, tal como es en la actualidad?.

\section{II.3. Objetivos del curso}

PROSODE, como curso, distingue dos niveles de objetivos. El primer nivel de objetivos está orientado hacia el aprendizaje y el desarrollo personal del estudiante, y a que la contribución que realicen nuestros alumnos hacia la comunidad se produzca de forma eficiente. En el desarrollo del curso, perseguimos que los estudiantes:

- experimenten una forma diferente de ejercer el Derecho: como un servicio a quien lo necesita;

- experimenten vivencias y/o comprensiones de la responsabilidad social universitaria y del abogado;

- conozcan y comprendan mejor la realidad nacional; y

- ejerzan roles en la realidad y anticipen, de esa manera, su futuro ejercicio profesional.

El segundo nivel está focalizado a los servicios brindados por nuestros alumnos a la población beneficiaria. Este nivel es el que hace posible identificar a PROSODE como una clínica jurídica. Se trata de materializar la finalidad de proyectarse en la sociedad, lo cual significa brindar servicios jurídicos a la comunidad y priorizar determinados sectores para facilitar el acceso a la justicia, a la defensa de los derechos humanos y a la formación ciudadana. Con todo ello, se contribuye a mejorar la calidad de vida de las personas.

\section{II.4. Modelo del curso}

Las siguientes son las principales características de nuestro curso:

- Es electivo (voluntario) y reconoce tres créditos.

- Puede ser llevado a partir de la mitad de la carrera.

- Es colectivo: comparte la metodología clínica, la del aprendizaje basado en problemas y la del aprendizaje-servicio ${ }^{10}$.

Ana Teresa Revilla, así como por estudiantes, quienes plantearon una propuesta que dio origen a PROSODE.

9 Para más información, puede revisarse la segunda parte del libro de ORTIZ, Iván (coordinador). Proyección social a través del Derecho y de PROSODE... Óp. cit.

10 Es colectivo, pues lo desarrollan tres profesores y ocho jefes práctica, quienes trabajan estrecha y coordinadamente con objetivos comunes tanto en la comunidad como en lo pedagógico. Esta metodología se desarrolló con el objetivo de mejorar la calidad de la educación médica al cambiar la orientación del currículo, que se basaba en una colección de exposiciones del maestro, hacia uno más integrado y organizado en problemas de la vida real, donde confluyen diferentes áreas del 
- Cuenta con una parte teórica y con otra práctica que genera dinamismo, pues los estudiantes actúan en la realidad.

- Se proyecta en la comunidad y facilita el acceso a la justicia, a la promoción y la defensa de derechos humanos, y a la formación ciudadana.

- Posee contenidos comunes en lo actitudinal, conceptual y procedimental (o habilidades). Adicionalmente, cada área cuenta con contenidos específicos.

- Se desarrolla dentro de la línea de docencia de la responsabilidad social universitaria, pues genera retorno de aprendizaje (actitudinal, habilidades y conocimientos) para nuestros estudiantes y docentes.

\section{II.5. Contenidos del curso}

Los contenidos comunes que se proponen para el aprendizaje de los estudiantes son los siguientes:

\section{II.5.1. Actitudinal}

El curso persigue desencadenar las siguientes actitudes comunes:

- intolerancia frente a la injusticia, a hechos o situaciones injustas;

- reconocimiento y preocupación por la existencia de personas cuyos derechos se encuentran en situación de vulnerabilidad (solidaridad);

- apoyo responsable a las personas que, víctimas de la injusticia, carecen de los medios para acceder a la defensa de sus derechos;

- preocupación, compromiso, responsabilidad y prudencia en sus intervenciones y acciones de consejo, apoyo y/o asesoría legal; y

- seguridad en su desenvolvimiento.

Por lo tanto, el curso promueve los valores de solidaridad, justicia, cultura de paz y ética en el desarrollo profesional.

\section{II.5.2. Contenido conceptual}

El curso promueve el desarrollo de contenidos temáticos comunes, seleccionados en función de las dificultades y de las necesidades fundamentales de la población a quien se dirige: Derecho civil, de familia,

PROYECCION

SOCIAL DE DERECHO: PROSODE,

UN APORTE DE

PROYECCIÓN Y

RESPONSABILI-

DAD SOCIAL 
derechos humanos, de niños y adolescentes, penal penitenciario y procesal civil.

\section{II.5.3. Contenido procedimental o habilidades}

Las habilidades comunes que pretendemos desarrollar son las siguientes:

- identificación de problemas socio-jurídicos;

- propuesta de alternativas de solución eficientes a situaciones jurídicas reales;

- investigación jurídica;

- análisis legal;

- trabajo en equipo;

- reconocimiento y resolución de dilemas éticos; y

- comunicación adecuada.

\section{II.6. Metodología}

En el curso, empleamos diversos métodos activos/colaborativos: el aprendizaje basado en problemas (ABP), el aprendizaje-servicio y, esencialmente, el método clínico.

\section{II.6.1. Aprendizaje basado en problemas ${ }^{11}$}

Nuestro curso cuenta con una parte práctica que implica la realización de actividades legales en beneficio de la comunidad como una fuente de enseñanza significativa y práctica aplicada a la solución de problemas reales. Así lo señala François Vallays ${ }^{12}$ : «Es preciso resaltar cómo los cursos de PROSODE (Proyección Social del Derecho) de la Facultad de Derecho de la PUCP constituyen un perfecto ejemplo de integración entre la docencia y el voluntariado con fines sociales, que puede servir de modelo para diseñar nuevas metodologías de enseñanza».

\section{II.6.2. Método clínico}

PROSODE no solo sigue métodos pedagógicos activos y colaborativos o casos y simulaciones, sino que constituye una clínica jurídica ${ }^{13}$ de interés particular. Nuestros alumnos actúan, en la realidad, en defensa de los derechos humanos y de los valores democráticos de personas de escasos recursos, y buscan una conexión entre la realidad social y jurídica.

Debemos señalar que las clínicas jurídicas y las acciones de proyección social de una facultad de Derecho se entrecruzan, pues, por esencia, toda clínica jurídica hace labor de proyección social universitaria en la

11 Es importante anotar que el curso fue presentado como una experiencia de ABP de nuestra universidad y de nuestra Facultad de Derecho en el Congreso Internacional de Aprendizaje Basado en Problemas organizado por la PUCP, durante 2006, en Lima.

12 VALLAEYS, François. «La responsabilidad social de la universidad». Óp. cit.

13 Es importante precisar que los profesores del curso han participado y/o organizado diferentes conferencias presenciales y por video, workshops y eventos sobre proyección social y clínica jurídica. 
comunidad. Sin embargo, no toda acción de proyección social emplea el método clínico. Asimismo, no todo método clínico es proyección social, pues cuando se realizan simulaciones no hay acción en la comunidad, sino un ejercicio o caso ficticio con un objetivo pedagógico.

Por ende, las clínicas jurídicas y la proyección social no son excluyentes sino complementarias: así, por un lado, la clínica jurídica ayudan a las facultades de Derecho a enseñar a los estudiantes cómo hacer el trabajo del abogado; y, por otro lado, también se pueden usar para ayudar a la sociedad, sea con casos de interés público o con casos individuales, como lo hace PROSODE a través de sus consultorios jurídicos gratuitos (asesoría legal) y del área penal-penitenciario.

\section{7. Áreas del curso}

El curso PROSODE se desarrolla a través de las siguientes áreas: asesoría legal, educación legal, difusión legal y penal-penitenciario.

\section{II.7.1. Asesoría legal}

Consiste en consultorías y seguimiento de casos en cinco consultorios jurídicos gratuitos. El alumno debe asumir el rol de abogado en casos reales, lo cual constituye un reto personal y académico.

En estos consultorios, los estudiantes entablan contacto con casos concretos, presentados directamente por los propios involucrados. Ello los motiva a participar directamente en la atención y en el seguimiento judicial de los procesos. Los alumnos tienen la oportunidad de iniciar su labor de futuros abogados, pues deben conocer el caso en su aspecto jurídico/legal y diseñar estrategias de defensa. Todo ello contribuye a la justicia sea un bien accesible a las personas.

\section{II.7.2. Educación legal}

El programa de educación legal está dirigido a promover la formación ciudadana y la defensa y la difusión de los derechos de niños y adolescentes. En los centros educativos, el estudiante de PROSODE transmite sus conocimientos de Derecho. Abarca una gama amplia de temas, como derechos de los niños y del adolescente y formación ciudadanía, entre otros más. A través de ellos, los alumnos experimentan problemáticas, viven y sufren como personas individuales y como integrantes de una familia. Se trata de un contacto muy productivo entre jóvenes, en el cual los temas de Derecho funcionan como catalizadores para la reflexión y para la conciencia del ejercicio de sus derechos.

\section{II.7.3. Difusión legal}

Consiste en la preparación, en la producción y en la emisión de programas socio-jurídicos radiales. Estos se transmiten una vez a la semana en una emisora a nivel nacional, y se reciben consultas al aire. 
Equipos de estudiantes aprenden a identificar los grandes problemas legales que aquejan a la población. A partir de su estudio y de investigaciones, los estudiantes deben poder elaborar un programa radial con los objetivos de difundir los derechos humanos y el cumplimiento de obligaciones, así como absolver consultas.

\section{II.7.4. Penal-penitenciario ${ }^{14}$}

Se realiza un trabajo legal en el penal San Pedro, ubicado en San Juan de Lurigancho. Se asume la defensa de internos de escasos recursos económicos y se pone en práctica la vocación de servicio y los conocimientos en Derecho penal. Se visitan establecimientos penitenciarios, juzgados y salas para conocer la realidad penitenciaria nacional y del sistema de justicia penal.

\section{IMPACTO EN LOS ESTUDIANTES}

¿El impacto de PROSODE en los estudiantes ha permitido el retorno en aprendizaje que sustenta la responsabilidad social universitaria? Es una pregunta difícil de contestar. Intentaremos responderla a través de indicadores que nos permitan observar impactos positivos del aprendizaje en los estudiantes que han cursado PROSODE.

En primer lugar, debemos preguntarnos si este curso ha convocado a los estudiantes durante estos diecinueve años de creado. El promedio de estudiantes matriculados desde su creación no ha sido de menos de cien anuales, sumados ambos semestres, con picos altos de más o menos 150 estudiantes matriculados en diferentes años. La convocatoria en general ha sido muy buena, por lo que podemos afirmar que este indicador es positivo.

En segundo lugar, la continuidad de estudiantes extranjeros de intercambio de universidades españolas que se repite en forma muy satisfactoria desde el año 2000 es un indicador claro de la receptividad y la aceptación de nuestra experiencia en el aprendizaje.

En tercer lugar, durante las reuniones presenciales de evaluación del curso y de la investigación que realizamos con los estudiantes en un período de cinco años ${ }^{15}$, brotan impresiones que son realmente muy importantes. Entre los principales aciertos del curso, tenemos los siguientes:

- Profesión como servicio

- Aprendizaje significativo del Derecho

14 Este trabajo es posible gracias a un convenio con el INPE ideado por el profesor Juan Luis Avendaño y realizado con el apoyo de Leonardo Caparrós, abogado de la PUCP y ex presidente del INPE, así como gracias al compromiso y la coordinación del doctor Iván Meini Méndez, coordinador del Área Penal del Departamento Académico de Derecho. La iniciativa fue impulsada en el marco de las celebraciones de los noventa años de la Facultad.

15 Para más información, puede revisarse la segunda parte del libro Proyección social a través del Derecho y de PROSODE... Óp. cit., pp. 225-256. 
- Visión integral de la profesión

- Desarrollo de habilidades/destrezas profesionales y no profesionales

- Profesión no solo como lucro

- Encuentro del perfil profesional

- Litigio

- Comprensión del significado de la injusticia en el país

- Conocimiento de la realidad

- Aprendizaje de conocimientos jurídicos

- Reconocimiento, redescubrimiento, profundización o cambio de valores, solidaridad, justicia y ética

De otro lado, aunque podríamos citar numerosas impresiones de los estudiantes, hemos escogido la siguiente, que resume el impacto en nuestros estudiantes ${ }^{16}$ :

Durante este ciclo mi experiencia en el curso de Proyección Social es y ha sido una de las más gratificantes a lo largo de mi experiencia estudiantil, puesto que con este curso aprendí que la educación es mucho más que un almacenamiento de conocimientos ordenados y estructurados. Es también la aplicación de estos a casos concretos; puesto que en el área de asesoría legal a la cual pertenecí pude poner en práctica mis conocimientos académicos a casos reales, a través de consultas, argumentación en escritos, seguimiento de los casos, etc. Adicionalmente, el lado de la responsabilidad y compromiso con los recurrentes hizo en mí una persona aun más organizada, y ni qué hablar del lado humanitario que se incentiva; pues hace posible tener una conexión de empatía y muchas veces de amistad con los recurrentes. Es así como este curso viabiliza el descubrimiento de las aptitudes de cada estudiante y la potenciación de aquellas actitudes que le servirán para desenvolverse en la sociedad. He aprendido tanto, y por ello siempre traté de dar mucho más. Me llevo grandes experiencias, pero sobre todo me llevo una enseñanza invaluable.

Por último, en las encuestas semestrales del curso, los resultados obtenidos han estado por encima del promedio de los cursos de la Facultad de Derecho. Las opiniones de los estudiantes sobre su aprendizaje han sido muy satisfactorias.

Por ello, observando estos indicadores, podemos resumir que el impacto en el aprendizaje de nuestros estudiantes ha sido altamente positivo y enriquecedor, lo que genera el retorno en aprendizaje de las acciones en la comunidad.

PROYECCION

SOCIAL DE DERE-

CHO: PROSODE,

UN APORTE DE

PROYECCIÓN Y

RESPONSABILI-

DAD SOCIAL 


\section{IMPACTO EN LA COMUNIDAD}

Solo para resumir el impacto de nuestro trabajo en la comunidad, durante 2009 hemos realizado las siguientes actividades por áreas:

- Asesoría legal. Se contaron 6399 atenciones, en nuestros cinco consultorios. De estas, 1548 constituyen casos nuevos. Un total de 569 procesos judiciales se encuentran en trámite.

- Difusión legal. Se realizaron 32 programas de El Derecho a tu Alcance en radio R-700, radio Milenia y radio La Luz. Se recibieron 221 llamadas, que hacen un promedio anual de 6,95 llamadas por programa.

- Educación legal. Se realizaron veintitrés talleres sobre derechos humanos dirigidos a los alumnos de quinto y sexto grado de primaria, y primero y segundo de secundaria, de los colegios Fe y Alegría $\mathrm{N}^{\circ}$ 04, ubicado en San Juan del Lurigancho; $\mathrm{N}^{\circ}$ 01, ubicado en San Martín de Porres; y Juan Pablo II, ubicado en Chorrillos. En 2009, se tuvo un total de 450 beneficiarios, entre niños, adolescentes y adultos.

- Penal-penitenciario. Se contó con la participación anual de ocho alumnos de la Facultad, quienes atendieron a internos del penal San Pedro en San Juan de Lurigancho. Se realizó el estudio a 23 casos de procesados y a 55 casos de sentenciados, un total de 78 casos. Es importante resaltar como logro haber conseguido la libertad de dos internos del penal de Lurigancho, así como el involucramiento de los estudiantes con el área de Derecho penal.

\section{A MODO DE CONCLUSIÓN}

La enseñanza del Derecho a través del curso Proyección Social - PROSODE busca que el alumno sea el protagonista de su aprendizaje significativo y que, a través de la acción en la realidad, utilice sus conocimientos y desarrolle habilidades jurídicas y no jurídicas bajo la supervisión de un profesor. Incluso, siendo más ambicioso, se pretende generar una formación integral y humanista, que permita nos contar, al concluir su formación, con abogados no solo profesionalmente idóneos sino, y sobre todo, con principios éticos.

Las principales dificultades de la justicia en nuestro país están vinculadas, entre otras, a problemas institucionales, de infraestructura, de desconocimiento del Derecho, de falta de confianza en el sistema y en la probidad de quienes la representan, así como en las limitaciones económicas de acceso a la justicia. Estos tres últimos aspectos se acentúan particularmente en los sectores pobres de nuestro país, cuando ello debe ser el requisito básico de un sistema legal orientado hacia la igualdad y la no discriminación. 
Partiendo de ambas premisas, podemos concluir que el curso de Proyección Social del Derecho - PROSODE viene fomentando, desde hace diecinueve años, la responsabilidad social universitaria. Facilita el acceso a la justicia y promueve la defensa de los derechos humanos al servicio de la comunidad. Esta se lleva a cabo a través de un curso voluntario de la Facultad de Derecho de la Pontificia Universidad Católica del Perú, con dos objetivos que se entrelazan: por un lado, lograr el aprendizaje de los estudiantes a través de la acción directa en la comunidad; por otro, que los alumnos brinden un servicio a las personas de escasos recursos económicos.

Por esta razón, pensamos afianzar una propuesta más integral en la Facultad, una de carácter obligatorio, que incluya una serie de opciones o alternativas que satisfagan los intereses de los alumnos sobre la base de lo existente, y la cual se sume la experiencia de PROSODE y de la clínica jurídica. Ello servirá para formar profesionales que sepan cómo ejercer su profesión, que pongan en práctica sus conocimientos y sus habilidades, y a quienes el contacto con la realidad les permita desarrollar habilidades, actitudes y principios éticos.

PROYECCION

SOCIAL DE DERE-

CHO: PROSODE,

UN APORTE DE

PROYECCIÓN Y

RESPONSABILI-

DAD SOCIAL 